\title{
A young man with oligoarthritis: what is his cardiovascular risk?
}

\author{
Emily Bolton, Kelsey Jordan and Karen Walker-Bone
}

\begin{abstract}
Gout is the commonest cause of inflammatory arthritis among young men. A case of acute gout in a 39-yearold man is described. As part of his assessment, his cardiovascular risk factors were evaluated. He was found to have elevated body mass index, central obesity, hypertension, a family history of cardiovascular disease, and hypercholesterolemia. This case highlights the association between gout,

hyperuricaemia and elevated cardiovascular risk. In young male patients, acute presentation with gout in primary care may provide the first opportunity for assessment of cardiovascular risk factors and primary prevention of ischaemic heart disease.

Keywords

general practice; gout; hyperuricaemia; risk factors, cardiovascular.
\end{abstract}

A 39-year-old male presented to his GP with acute pain and swelling in his left ankle and knee, which had developed over the preceding 24 hours. The patient described a 14-year history of flitting arthritis, usually mono-arthritis, lasting initially 12-24 hours. Over time, he had experienced multiple recurrences occurring at progressively shorter intervals. At the time of presentation, he was experiencing episodes monthly, lasting approximately 10 days each time. Affected joints at different times included the hands, wrists, elbows, knees, ankles, and halluxes. Up until now, the patient had managed these episodes with rest, paracetamol and diclofenac (prescribed originally for mechanical back pain) as required. He had no family history of arthritis. He was a nonsmoker and drank 4 units of alcohol weekly. The GP prescribed tramadol and omeprazole because of the diclofenac, and referred the patient to rheumatology urgently.

On examination in rheumatology, the patient weighed $119.40 \mathrm{~kg}$ (body mass index $35.7 \mathrm{~kg} / \mathrm{m}^{2}$ ) and his blood pressure was $155 / 102 \mathrm{mmHg}$. He was abdominally obese. He was walking with difficulty and could not wear outdoor shoes. Musculoskeletal examination revealed a small effusion in the left wrist, normal hands, elbows, shoulders, hips, and spine, but large effusions in his left ankle and knee, associated with significant reduction in range of movement. His right hallux had severe valgus deformity associated with swelling.

Investigation showed an erythrocyte sedimentation rate (ESR) of $39 \mathrm{~mm} / \mathrm{hour}$ and Creactive protein (CRP) $66 \mathrm{mg} / \mathrm{l}$, normal white cell count, and elevated uric acid $0.61 \mu \mathrm{mol} / \mathrm{l}$ (normal range $0.2-0.42 \mu \mathrm{mol} / \mathrm{l})$. Large quantities of needleshaped crystals were seen in the knee joint aspirate, which were negatively birefringent under polarising light microscopy. A diagnosis of gout was established. ${ }^{1}$ As part of the management, he was screened for cardiovascular risk factors. His urine dipstick was negative for protein and glucose. His fasting lipid profile showed a high fasting cholesterol of $6.8 \mathrm{mmol} / \mathrm{l}$ (normal range $2.08-5.0 \mathrm{mmol} / \mathrm{l}$ ), an increase in low-density lipoprotein (LDL) cholesterol to $4.9 \mathrm{mmol} / \mathrm{l}$ (normal $<3 \mathrm{mmol} / \mathrm{l}$ ), and elevated plasma cholesterol:high-density lipoprotein (HDL) ratio of 4.9. Fasting glucose was normal. His paternal grandfather had died of a myocardial infarction at the 


\section{How this fits in}

GPs are familiar with the diagnosis and management of gout. However, this

case illustrates the important relationship between hyperuricaemia and other risk factors for the metabolic syndrome and cardiovascular disease. In young men, gout is the commonest type of inflammatory arthritis and as this

demographic group are infrequent attendees in primary care, it may provide one of few opportunities to recognise and respond preventively to elevated cardiovascular risk. It is currently unclear whether hyperuricaemia is itself a direct cardiovascular risk factor or is elevated as part of a pathway. However, allopurinol which lowers urate through its uricosuric properties has been shown to have antihypertensive effects and may also have beneficial effects on cardiovascular disease through reduction of oxidative stress.

age of 55 years and his mother had been diagnosed with type 2 diabetes mellitus recently before presentation. Based on age, systolic blood pressure, total cholesterol, HDL, blood pressure therapy, diabetes, and sex, a 10-year risk of myocardial infarction for this patient was estimated to be $9.5 \%{ }^{2}$

Gout is a disorder of purine metabolism characterised by acute, recurrent attacks of crystal arthritis. The prevalence of gout in the UK population has been estimated at $1.4 \%$, increases with age, and is much higher among men. ${ }^{3,4}$ The most important risk factor is elevated serum uric acid, with supersaturation of uric acid in the extracellular fluid causing precipitation of uric acid crystals. Deposition of crystals in the articular, periarticular, and subcutaneous tissues results in episodes of acute arthritis and tophus development.

\section{URIC ACID AND CARDIOVASCULAR RISK}

As long ago as the late 19th century, researchers described an association of gout with hypertension, diabetes, kidney disease, and cardiovascular disease. $^{5}$ More evidence for this association was obtained from epidemiological studies in the mid20th century which demonstrated associations between hyperuricaemia and a variety of cardiovascular diseases (hypertension, metabolic syndrome, coronary artery disease, cerebrovascular disease, vascular dementia, and pre-eclampsia). ${ }^{5}$ Moreover, relationships between uric acid and cardiovascular diseases were not only seen in the range of frank hyperuricaemia $(360 \mu \mathrm{mol} / \mathrm{l}$ in females and $420 \mu \mathrm{mol} / \mathrm{l}$ in males) but also with levels of uric acid at the upper end of the normal range (310-330 $\mu \mathrm{mol} / \mathrm{l})$.

There has been much controversy as to whether hyperuricaemia is itself an independent risk factor for cardiovascular disease. ${ }^{6}$ Until now, the weight of evidence has favoured that hyperuricaemia is an incidental risk factor and that clinicians should rely on assessment and treatment of traditional Framingham risk factors (smoking, hyperlipidaemia, diabetes, and hypertension). Hyperuricaemia is associated with comorbidities that are themselves cardiovascular risk factors: a recent European study showed that obesity was the most common comorbidity (prevalence 27.7\%) in the UK, whereas diabetes was the most frequent comorbidity in Germany (prevalence 25.9\%). ${ }^{4}$ Hypertension was seen in $17.5 \%$ and heart failure and myocardial infarction were reported in $7.1 \%$ and $7.4 \%$ of UK patients. The prevalence of cardiovascular comorbidities increased with serum uric acid level. ${ }^{4}$

Recently, new evidence from animal, clinical, and epidemiological studies has led to a re-appraisal of the association between serum urate and cardiovascular disease. ${ }^{5,6}$ In epidemiological studies, increasing urate has been associated with subsequent development of hypertension, ${ }^{5}$ and in small clinical studies, treatment with the uratelowering therapy allopurinol reduced systolic and diastolic blood pressure among patients with stage 1 hypertension who were naive to antihypertensive drugs. Urate levels have also been associated with surrogate markers of atherosclerosis, such as intima-media thickness ${ }^{4,5}$ and arterial stiffness. Allopurinol may have a role in reduction of oxidative stress, ${ }^{7}$ and it is possible that the positive effect of this drug on hypertension is mediated through this mechanism.

Whether hyperuricaemia is causally associated itself or acting as a marker of other associated cardiovascular system risk factors, this case highlights that all patients presenting with acute gout are at significantly increased risk of cardiovascular disease. As with this patient, young males are infrequent consulters in primary care and, in this case, it is contested that this patient's extreme cardiovascular risk was only identified opportunistically when he attended because of joint pain.

Finally, gout is a common condition in primary care, which can be acutely painful, can become a chronic disabling condition, and is associated with comorbidities including cardiovascular disease. Gout therapies are available that are capable of reducing serum urate and improving quality of life. Audits suggest that management is variable and quite frequently poor in clinical practice.

The British Society of Rheumatologists has drawn up evidence-based guidelines for the management of gout, which include advice about diet and lifestyle modification, including alcohol intake, as well as appropriate use of drug therapies. ${ }^{8}$ These guidelines recommend assessment and treatment of cardiovascular risk factors. Based on the available 
evidence, these guidelines suggest that physicians should aim to maintain plasma urate below $300 \mu \mathrm{mol} / \mathrm{l}$ for optimal care. ${ }^{8}$ Therefore it is concluded that in a patient with asymmetric oligoarthritis, the risk of cardiovascular disease may be substantially elevated.

\section{Consent}

The patient gave consent for publication of this case history.

\section{Competing interests}

The authors have stated that there are none.

\section{Discuss this article}

Contribute and read comments about this article on the Discussion Forum: http://www.rcgp.org.uk/bjgp-discuss

\section{REFERENCES}

1. Zhang W, Doherty M, Pascual E, et al. EULAR evidence based recommendations for gout. Part I: Diagnosis. Report of a task force of the standing committee for international clinical studies including therapeutics (ESCISIT). Ann Rheum Dis 2006; 65(10): 1301-1311.

2. D'Agostino RB, Vasan RS, Pencina MJ, et al. General cardiovascular risk profile for use in primary care: The Framingham Heart Study. Circulation 2008; 117(6): 743-753.

3. Mikuls TR, Farrar JT, Bilker WB, et al. Gout epidemiology: results from the UK General Practice Research database, 1990-1999. Ann Rheum Dis 2005; 64(2): 267-272.

4. Annemans L, Spaepen E, Gaskin M, et al. Gout in the UK and Germany: prevalence, comorbidities and management in general practice 200-2005. Ann Rheum Dis 2008; 67(7): 960-966.

5. Feig DI, Kang D-H, Johnson RJ. Uric acid and cardiovascular risk. $N$ Engl J Med 2008; 359(17): 1811-1821.

6. Gaffo AL, Edwards NL, Saag KG. Gout. Hyperuricaemia and cardiovascular disease: how strong is the evidence for a causal link? Arthritis Res Ther 2009; 11(4): 240-247.

7. Corry DB, Eslami P, Yamamoto K, et al. Uric acid stimulates vascular smooth muscle cell proliferation and oxidative stress via the vascular rennin-angiotensin system. J Hypertens 2008; 26(2): 269-275.

8. Jordan KM, Cameron JS, Snaith M, et al. British Society for Rheumatology and British Health Professionals in Rheumatology guidelines for the management of gout. Rheumatology 2007; 46(8): 1372-1374. 